\title{
ERS Transform for the Detection of Bronchi on CT of the Lungs
}

\author{
François Chabat, David M. Hansell, and Guang-Zhong Yang \\ Imaging Department. Royal Brompton Hospital. Sydney Street. \\ London SW3 6NP. UK \\ f. chabaterbh.nthames.nhs.uk
}

\begin{abstract}
The identification of bronchi on Computed Tomography (CT) images of the lungs provides valuable clinical information for the assessment of patients with suspected bronchiectasis, emphysema, or constrictive obliterative bronchiolitis. The automated recognition of the airways is an important part of a diagnosis-aid system. It resolves potential ambiguities associated with intensitybased feature extractors. On CT images, cross-sections of bronchi normally appear as elliptical rings and this paper presents a novel technique for their recognition. The proposed method, the ERS transform, is based on the analysis of the distribution of edges in local polar co-ordinates. Pixels are ranked according to local edge (E) strength, radial $(R)$ uniformity. and local symmetry (S). A discrete implementation of the technique is provided which reduces the computational cost of the ERS transform by using a geometric approximation of the intensity patterns. The method compares favourably to other methods such as template matching or Hough transform. Noise-sensitivity of the technique was evaluated on a set of synthetic images and patient study was undertaken with a set of 27 cross-sectional images showing different lung pathologies. Agreement with an experienced radiologist was reached in 76 out of 136 bronchi (agreement rate: $57 \%$ ), which suggests satisfactory statistical significance for using the ERS transform as part of a computerised diagnosis aid system.
\end{abstract}

\section{Introduction}

Computed Tomography (CT) is a valuable imaging modality for assessing lung diseases such as bronchiectasis, emphysema, or constrictive obliterative bronchiolitis. From a set of cross-sectional images of the lungs, experienced radiologists achieve differential diagnosis by taking into account CT findings such as the density differences of the lung parenchyma, the size and distribution of the pulmonary vessels, and the state of the bronchi. For the detection of small airways disease, the most important finding is the identification of areas of decreased attenuation in the lung parenchyma. Based on global intensity distribution, we have developed several methods for the quantification of the extent of under-attenuated areas of the lungs [1][2][3]. The inherent problem with these methods is that the contextual information is not taken into account. Indeed, dark patches in the lung parenchyma cannot be confidently labelled as abnormal without ancillary findings, such as the morphology 
of the pulmonary vessels and the airways. Several low level feature extractors need to be combined in a high-level system incorporating a priori knowledge to achieve computerised diagnosis aid. Within this framework, essential clinical information can be derived from the morphology of the airways by measuring bronchial dilation and wall thickening. As an example, the presence of intensity differences in the lung parenchyma (known as mosaic attenuation pattern) can be caused by different pathologies: interstitial lung disease, vascular abnormalities, or airway disease. The identification of bronchial wall dilation on inspiratory scans suggests an airway disease rather than a vascular cause. This example stresses that there are ambiguities associated with the sole consideration of intensity differences. To resolve them and derive clinically meaningful conclusions, an algorithm for the automated detection of bronchi is necessary.

On cross-sectional images, bronchi rumning near perpendicular to the image plane appear as bright elliptical rings. This paper presents a novel technique for the recognition of elliptical rings, based on their geometric properties. An Edge-RadiusSymmetry (ERS) transform is used for the analysis of gradient maxima and minima in local polar co-ordinates. Based on the intensity (measured by function $E$ ), radial distribution (measured by function $R$ ), and symmetry (measured by function $S$ ) of these maxima and minima, the pixels are ranked to provide a sorted list of the most likely positions of all dominant elliptical rings. The algorithm was evaluated on synthetic and patient data showing different lung pathologies.

\section{Background}

Along a short distance. bronchi can be assimilated to cylindrical structures. They are filled with air and thus the luminal CT density is of the value of - 1000 Hounsfield Units (H.U.). The bronchial wall consists of tissues of higher density (typically 100 H.U.). On CT images, perpendicular cross-sections of bronchi therefore appear as circular bright rings superimposed on the lung parenchyma. which has a low average CT density (typically $-900 \mathrm{H} . \mathrm{U}$.). When the plane of acquisition is not precisely

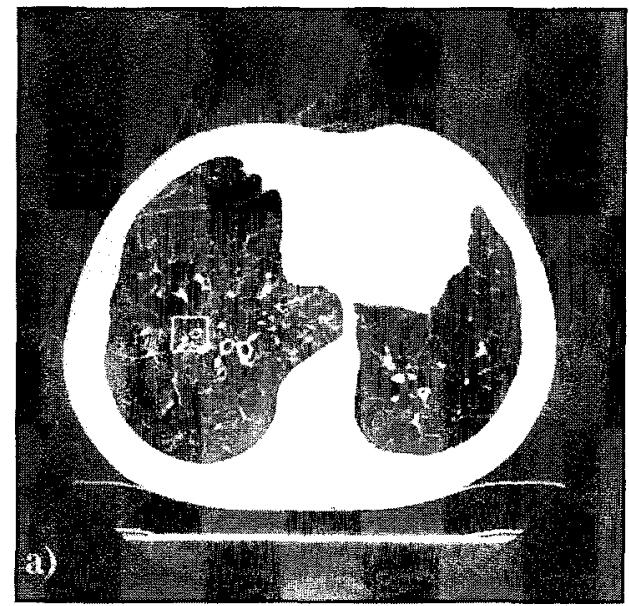

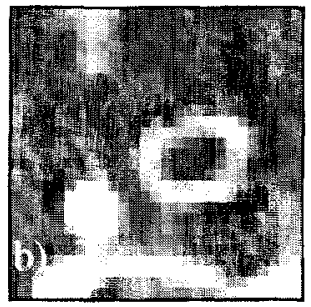

Fig. 1. a) Example of an HRCT scan of the lungs (window settings: level $=-800 \mathrm{H} . \mathrm{U}$. $/$ width $=1000 \mathrm{H} . \mathrm{L} . \mathrm{S}$. Bronchi perpendicular to the plane of acquisition appear as bright rings. The white square highlights the position of a major bronchus. b) Magnified view of the major bronchus highlighted in Figure (1b). 
perpendicular to the main axis of a bronchus, the bronchial wall appears as an elliptical ring. Figure (1) provides an example of High-Resolution Computed Tomography (HRCT) scan of the lungs, with the conspicuous near-perpendicular cross-section of a major airway, which can be identified as a bright elliptical ring. Because of partial volume effect and the limits in image resolution, small bronchi may be partially hidden and difficult to detect.

There is little work reported in the literature for the automated identification of bronchi on CT images of the lungs, but semi-automated techniques requiring user interaction have been described [4]. This may be due to the difficulty for automated methods to match the subjective assessment made by experienced radiologists. Indeed. it has been demonstrated that inter-observer agreement for the detection of bronchial wall thickening, for instance, is consistent (kappa value $\kappa=0,6$ ) [5]. Still, to incorporate contextual information into a diagnosis aid system based firstly on the analysis of intensity differences [1], it is necessary to design an automated technique for the detection of bronchi. The transform presented in this paper is a necessary tool meant to be used in conjunction with other features extractors, combined in a highlevel framework to resolve local ambiguities.

\section{Automated pattern identification}

Template matching and Hough transform are common for the detection of circular patterns. Although they are suitable for the current application of detecting bronchial wall, they can be computationally expensive due to the high number of parameters required to define a ring-shaped structure. These parameters include position. size, orientation, ratio of the short and long axes. and width of the ring. The five parameters involved for detecting an ellipse, for instance, can make a standard Hough transform impractical in terms of computational and memory demands and even adaptive and two-pass methods remain time consuming [6].

\subsection{ERS Transform}

The ERS transform introduced in this paper is based on the analysis of the distribution of gradient maxima and minima in the neighbourhood of each pixel. Ellipses and rings are patterns symmetrical relatively to their centroids. In polar co-ordinates, the expression of the intensity and spatial distribution of the most significant edges has characteristic properties of symmetry and uniformity. The ERS transform is based on the analysis of edge distribution in local polar co-ordinates. In the case of CT images, given the CT densities of air, bronchial wall, and lung parenchyma, the intensity gradient should show local maxima and minima at the points on the inner border (i.e. at the interface between airway and bronchial wall) and outer border (i.e. at the interface between bronchial wall and lung parenchyma) of a bronchus respectively. Let $I(x, y)$ be the original image, we consider the local polar co-ordinates $(r, \theta)$ centred at $\left(x_{o}, y_{0}\right)$, and a neighbourhood $\Omega$ of radius $r_{o}$. The maximum of the intensity gradient within $\Omega$ along direction $\theta$ is defined as $e_{l}(\theta)$ : 


$$
e_{1}(\theta)=\max _{0<r<r}\left(\frac{\partial I}{\partial r}(r, \theta)\right)
$$

with its corresponding radius defined as $r_{2}(\theta)$. The size $r_{0}$ of the neighbourhood $\Omega$ is to be defined according to the resolution of the image and the typical size of the patterns to identify. Similarly, the minimum of the intensity gradient along direction $\theta$ within the neighbourhood $\Omega$ is defined as $e_{2}(\theta)$ :

$$
e_{2}(\theta)=\min _{0 \ltimes r<r}\left(\frac{\partial I}{\partial r}(r, \theta)\right)
$$

and its radius is denoted as $r_{2}(\theta)$. This generates four functions in relation to $\theta: e_{f}(\theta)$, $e_{2}(\theta), r_{i}(\theta)$, and $r_{2}(\theta)$ which describe the radial distribution of edges around point $\left(x_{0}\right.$. $\left.y_{0}\right)$. Figure (2) gives a schematic representation of the definition of $e_{i}(\theta), e_{2}(\theta), r_{i}(\theta)$, and $r_{2}(\theta)$ for a pixel $\left(x_{\theta} y_{0}\right)$ located inside an elliptical ring, shown in Figure (2a). By searching along direction $d_{\theta}$, the gradient maximum $e_{1}(\theta)$, shown in the gradient profile in Figure ( $2 b)$, is found at the inner border of the ring. Similarly, the gradient minimum $e_{2}(\theta)$ is found at the outer border of the ring. The positions $r_{i}(\theta)$ and $r_{2}(\theta)$ of these maximum and minimum, relatively to $\left(x_{\theta}, y_{0}\right)$, provide the intersection points of the ring along direction $d_{\theta}$.

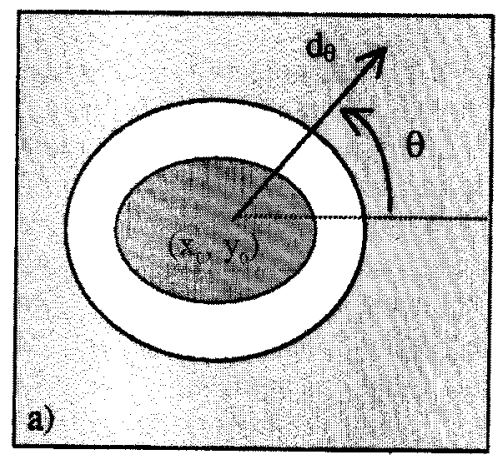

b)

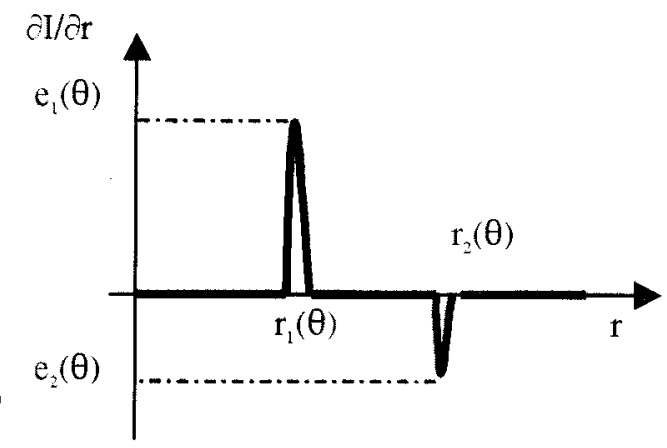

Fig. 2. a) Schematic representation of the function $I(x, y)$ for an elliptical ring. The darkest areas represent the lowest intensities. At pixel $\left(x_{\theta}, y_{0}\right)$, the maximum and minimum of the intensity gradient are searched along each direction $d_{\theta}$. b) The profile of the intensity gradient along direction $d_{\theta}$. The distance to the pixel $\left(x_{r}, y_{\theta}\right)$ is denoted $r$, and $d / c r$ measures the intensity gradient. At the inner border of the ring, a sharp intensity increase results in a maximum peak $e_{1}(\theta)$ at a distance $r(\theta)$. Further, at the outer border of the ring. a sharp intensity decrease results in a minimum peak $e_{(}(\theta)$ at a distance $r_{2}(\theta)$.

At every pixel $(x, y)$, three quantities $E, R$, and $S$ are then derived from the functions $e_{1}(\theta), e_{2}(\theta), r_{i}(\theta)$, and $r_{2}(\theta)$.

- The value $E(x, y)$ measures the strength of the edges found in the neighbourhood of $(x, y)$. It is defined as: 


$$
E(x, y)=\frac{1}{\operatorname{mean}_{0 \leq \theta<2 \pi}\left(\left|e_{1}(\theta)\right|\right)+\operatorname{mean}_{0 \leq \theta<2 \pi}\left(\left|e_{2}(\theta)\right|\right)}
$$

For a well-defined ring with sharp edges, the values of $e_{j}(\theta)$ are highly positive and the values of $e_{2}(\theta)$ are highly negative, making $E(x, y)$ small.

- The value $R(x, y)$ measures the uniformity of the radial distribution of the edges. It can be computed as:

$$
R(x, y)=\sigma\left(r_{1}(\theta)\right)+\sigma\left(r_{2}(\theta)\right)
$$

where $\sigma(f(\theta))$ is the average deviation of $f(\theta)$.

For a perfectly circular ring centred at $(x, y)$, the values of $r_{i}(\theta)$ are all identical, and so are the values of $r_{2}(\theta)$, yielding to a minimal value of $R(x, y)$ of zero.

- The value $S(x, y)$ measures the symmetry of the strength of the edges. It is defined as:

$$
S(x, y)=\underset{0 \leq \theta<2 \pi}{\operatorname{mean}}\left(\delta_{1}(\theta)\right)+\operatorname{mean}_{0 \leq \theta<2 \pi}\left(\delta_{2}(\theta)\right)
$$

where:

$$
\delta_{j}(\theta)=\frac{\max \left(e_{j}(\theta), e_{j}(\theta+\pi)\right)}{\min \left(e_{j}(\theta), e_{j}(\theta+\pi)\right)}-1
$$

The value of $\delta(\theta)$ measures the difference of contrast between two opposing edges relatively to $(x, y)$. For a pattern that is perfectly symmetrical in relation to $(x, y)$, the function $\delta(\theta)$ is always nil, yielding to a minimum value $S(x, y)$ of zero.

If $(x, y)$ denotes the location of a well-defined elliptical ring, the values $E(x, y), R(x, y)$ and $S(x, y)$ should therefore be small. The ERS transform is performed by combining numerically these three measures. Each pixel is given a rank $n_{E}$ reflecting its position in the list of pixels sorted according to $E(x, y)$. The pixel with rank $n_{E}=1$ represents the location with the lowest value of $E(x, y)$ (i.e. the sharpest edges) found on the image. Similarly, each pixel is given a rank $n_{R}$ and a rank $n_{s}$ according to its value of $R(x, y)$ and $S(x, y)$ respectively. The three ranks are then combined to provide a list of pixels sorted according to the value of $n$ defined as:

$$
n=n_{E}+n_{R}+n_{s}
$$

The pixel with the highest rank $n$ represents the position of the most dominant elliptical ring within the image. This ranking mechanism ensures that there is no need to normalise and weight the three quantities $E(x, y), R(x, y)$ and $S(x, y)$. Since pixels are sorted according to each of the criteria independently, the final ranking does not depend on how rapidly the numerical expressions of $E, R$, and $S$ increase. 


\subsection{Discrete approximation}

Since the typical size of the elliptical patterns to be identified is less than 12 pixels, it is possible to derive a precise approximation of them by considering only 8 points, taken along 8 principal directions, rather than considering all points of an ellipse. The 8 directions $\left\{d_{i}\right\}_{1 \leq i \leq 8}$ are taken along the horizontal, vertical and principal diagonal axes, and are indexed clockwise consecutively. Edge information is derived from the original image by pre-computing the gradient along directions $d_{1}, d_{2}, d_{3}$, and $d_{4}$. The intensity gradient maxima and minima along directions $\left\{d_{i}\right\}_{1 s_{i} \leq s}$ are represented by 8 -

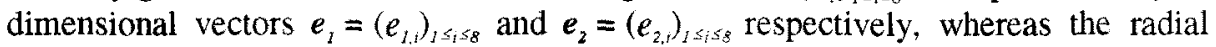
distribution of the edges are represented by vectors $r_{1}=\left(r_{t, i}\right)_{1 s_{i} \leqslant 8}$ and $r_{2}=\left(r_{2, i}\right)_{1 s_{i} \leq g}$. The definitions of $E(x, y), R(x, y)$ and $S(x, y)$ are the discrete equivalent of those given in equations and (3), (4) and (5).

In the discrete implementation of the ERS transform, the ranking procedure involved is done using the quicksort algorithm. On average, the computational cost of the discrete implementation of the ERS transform is $O\left(N \log N, r_{0}\right)$, with $N$ being the number of pixels, and $r_{i}$ being the radius of the neighbourhood searched at each point.

As an example, Figure (3) demonstrates the pixels ranked according to $E, R$, and $S$ for a synthetic image. Figure (3a) represents an image with elliptical rings and nonelliptical patterns with different sizes. The arrow highlights incomplete rings and partly occluded patterns. The SNR of this image is $44 \mathrm{~dB}$, which is close to that of a typical HRCT scan. The locations surrounded by significant edges have high ranks $n_{E}$ as shown in Figure ( $3 b$ ), signified by the brightness of the pixels. The results for $R(x, y)$ (uniformity) and $S(x, y)$ (symmetry) are illustrated in Figures (3c) and (3d) respectively. The incomplete patterns highlighted by the arrow on Figure ( $3 a$ ) are penalised by low ranks $n_{5}$, as shown in Figure (3d).

\section{Results}

\subsection{Noise sensitivity}

Since the proposed method is effectively based on the analysis of local maxima and minima of the first derivatives of the image intensity, it is necessary to evaluate its sensitivity to noise. A synthetic image, shown in Figure (3a), displaying various elliptical rings with different sizes was created to evaluate the noise-sensitivity of the edge-based technique. The intensity of the patterns is typical of the structures seen on HRCT scans of the lungs. The added Gaussian noise is similar to the noise inherent to the imaging modality. In the first 100 locations found by the algorithm, no false positive was detected, and all rings were identified. 

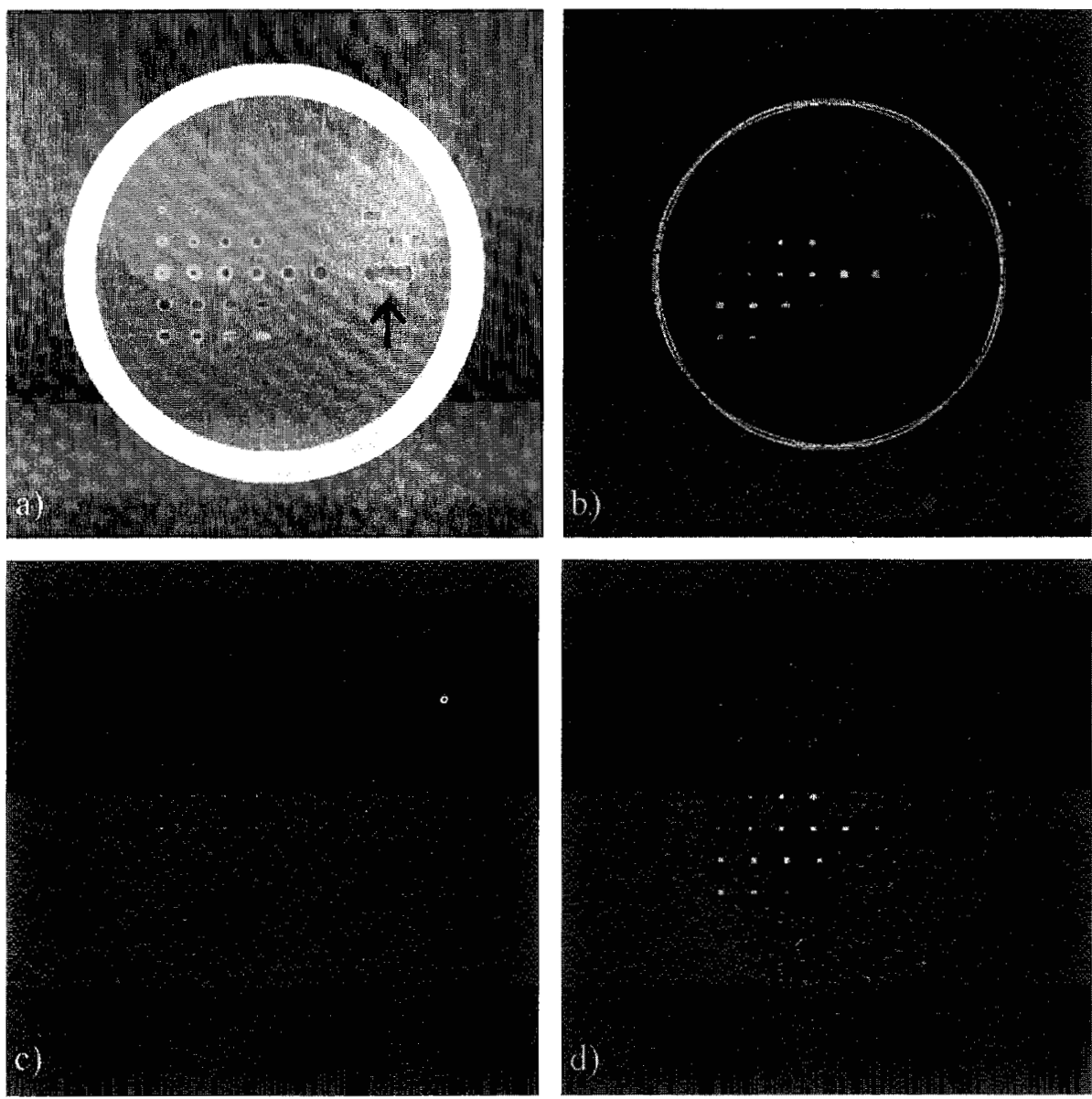

Fig. 3. a) Original synthetic image showing various elliptical and non-elliptical patterns with different sizes. The arrow highlights incomplete and partly occluded patterns. Gaussian noise was superimposed to the image $(\mathrm{SNR}=44 \mathrm{~dB})$. b) Ranks $n_{\bar{\varepsilon}}$ derived from $E(x, y)$ (displayed only in the large inner circle). Pixels surrounded by strong edges have high ranks $n_{z}$ and appear brighter. c) Ranks $n_{\xi}$ derived from $R(x, y)$. The centres of the elliptical patterns are highlighted. $d)$ Ranks $n_{s}$ derived from $S(x, y)$. The partly occluded patterns have poor edge strength symmetry and appear darker.

Measuring how the method copes with added Gaussian noise of higher variance shows that, for a signal-to-noise ratio (SNR) equal to or lower than $14 \mathrm{~dB}$, no false positive is found amongst the first 100 results. With an SNR equal to $12 \mathrm{~dB}, 3 \%$ of false negatives and $4 \%$ of false positive can be found in the first 100 results. 


\subsection{Patient studies}

To assess the value of the method in a clinical context, the ERS transform was applied to CT scans of the lungs, which were also scored by an experienced radiologist. For 9 patients presenting suspected airways disease, 3 HRCT scans $(1.5 \mathrm{~mm}$ collimation, sharp kernel reconstruction algorithm) were acquired at 3 pre-defined anatomical level in the upper, mid and lower zones of the lungs respectively. A radiologist identified on each of these 27 cross-sectional images the positions of the bronchi he regarded as most significant in a clinical evaluation. Because of the documented high inter-observer agreement for the assessment of bronchi on HRCT scans [5], a single human observer was considered reliable. The ERS transform was also applied and, for each scan, the list of the $N$ first positions estimated by the algorithm was compared to the $N$ positions determined by the human observer ( $N$ being case-dependent, and fixed for each case by the radiologist). Each location found both in the $N$-long list established by the radiologist and the $N$-long list established by the automated method was regarded as a case of agreement.

On a set of 27 cross-sectional images, the radiologist found 136 significant bronchi. Agreement with the ERS transform was reached in 76 instances, giving a rate of agreement of $57 \%$. Figure (4) shows an example of the compared results found by the human observer, as displayed by the crosses on Figure (4a), and the proposed algorithm, as displayed by the crosses on Figure ( $4 \mathrm{~b})$. In this case, agreement was reached in 9 out of 10 instances.

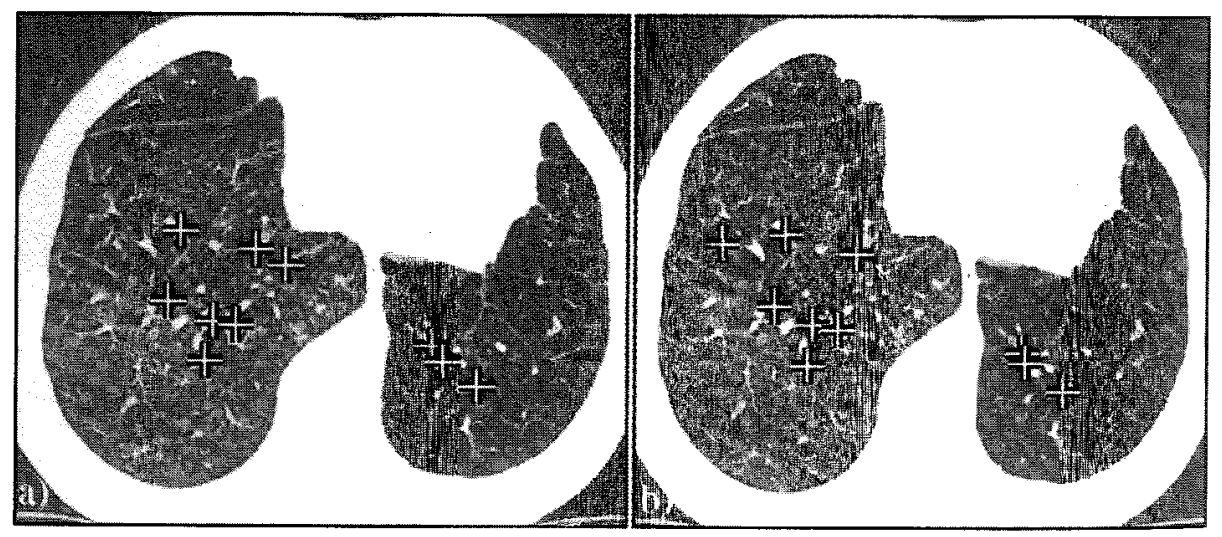

Fig. 4. a) HRCT scan of the lower zone of the lungs. The most significant bronchi. as identified by an experienced radiologist. are marked with a cross. b) Bronchi found by the ERS transform on the same image. The automated method matches the results of the human observer in 9 out of 10 instances.

Figure (5) shows an example of HRCT scan yielding to strong disagreement between the human observer and the automated method. The bronchi that the radiologist highlights for their clinical value, shown on Figure (5a), may not have the most clearly defined contours. Therefore, agreement with ERS transform cannot be reached, as shown in Figure $(5 b)$. However, by reading Figure $(5 b)$ retrospectively, it 
appears that the patterns identified by the ERS transform are indeed elliptical rings, in accordance with the expected behaviour of the algorithm.

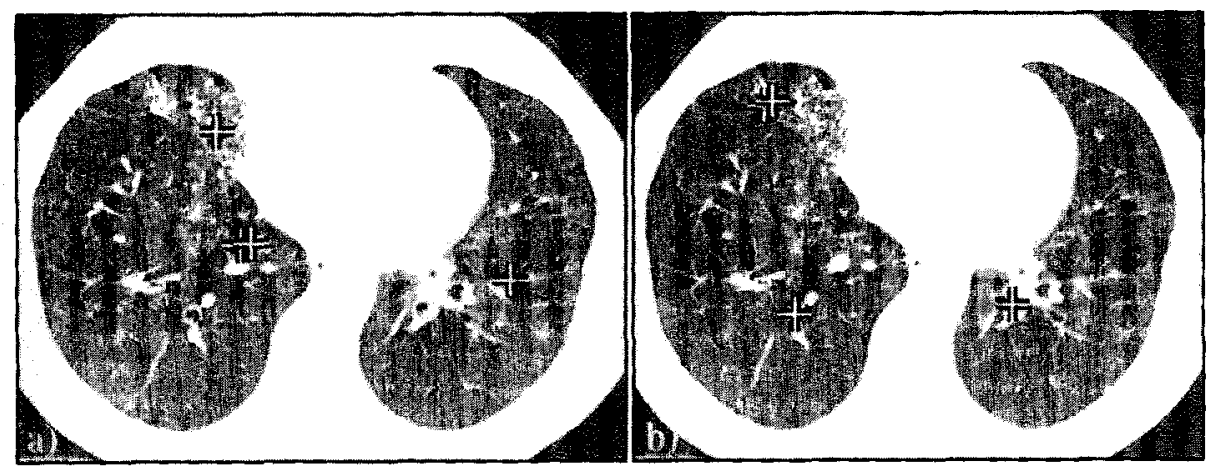

Fig. 5. Example of an HRCT scan with strong disagreement between the human and automated assessment. a) Airways identified by the human observer. The bronchi retained for the clinical information they provide are not necessarily the best-defined patterns with the strongest edges. b) ERS transform. Elliptical rings are detected but they were not selected by the radiologist for their clinical value.

\section{Discussion}

The elliptical rings to identify being defined by 6 parameters, the computational cost of methods like template matching and Hough transform (complexity $O\left(N^{i}\right)$ ) is considerable [7]. The implementation of the ERS transform compares favourably with these techniques.

The agreement rate of $57 \%$ is satisfactory for a low-level feature extractor such as the ERS transform. The patient study showed that the bronchi identified by the algorithm presented the expected geometric properties. When the human observer did not primarily choose them, it was due to high-level clinical knowledge. For example, the structures identified within consolidated lung or at a level near bifurcation, as shown in Figure $(5 b)$, are indeed bronchi, but are discarded by an experienced observer because the reliable interpretation of airways in these situations is problematic. This radiological expertise is out of the scope of the proposed algorithm. However, using the ERS transform, detection of bronchi is still possible. In a computerised diagnosisaid system, multiple feature extractors are used. Their outputs are combined in a statistical high-level framework incorporating a priori knowledge, in order to reach valid clinical conclusions. In that context, the agreement rate of $57 \%$ between the proposed method and a human observer suggests satisfactory statistical significance. 


\section{Conclusion}

In this paper, we have presented a novel technique for the detection of elliptical rings. The ERS transform is based on edge analysis in local polar co-ordinates, and relies on the geometric properties of the patterns to identify. It was shown to cope robustly with noise. Application to the detection of bronchi on HRCT scans of the lungs was also demonstrated. Comparison with the assessment made by a human observer showed an agreement rate of $57 \%$, allowing for the use of the proposed method in a computerised diagnosis aid system.

\section{References}

[1] Chabat F, Hansell DM, Yang GZ, CT Lung image classification with correction for perfusion gradient. in Proceedings of the Seventh IEE Conference on Image Processing and Applications; 1999. In press.

[2] Yang GZ, Chabat F. Hansell DM. Enhancement of subtle intensity differences of the lung parenchyma on CT. British Journal of Radiology; 1998, 71(846):686-90

[3] Yang GZ, Hansell DM. CT Image enhancement with wavelet analysis for the detection of small airways disease. IEEE Transactions on Medical Imaging: 1997 , 6(16):953-961.

[4] Senéterre E. Paganin F, Bruel JM, Michel FB. Bousquet J. Measurement of the internal size of bronchi using high resolution computed tomography (HRCT). Eur Respir J; 1994, 7: 596-600.

[5] Reiff DB, Wells AU, Carr DH, Cole PJ, Hansell DM. CT Findings in bronchiectasis: limited value in distinguishing between idiopathic and specific types. AJR; 1995, 165:261-267.

[6] Yuen HK, Illingworth J, Kittler J. Detecting partially occluded ellipses using the Hough transform, Image and Vision Computing; 1989, vol. 7, 1:31-37.

[7] Haralick RM, Shapiro LG. Computer and Robot Vision. Volume 1. AdudisonWesley publishing company, 1992. 